\title{
Spectral flow of trimer states of two heavy impurities and one light condensed boson
}

\author{
N. T. Zinner \\ Department of Physics and Astronomy, Aarhus University, Aarhus C, DK-8000
}

August 28, 2021

\begin{abstract}
The spectral flow of three-body (trimer) states consisting of two heavy (impurity) particles sitting in a condensate of light bosons is considered. Assuming that the condensate is weakly interacting and that an impurity and a boson have a resonant zero-range two-body interaction, we use the Born-Oppenheimer approximation to determine the effective three-body potential. We solve the resulting Schrödinger equation numerically and determine the trimer binding energies as a function of the coherence length of the light bosonic condensate particles. The binding energy is found to be suppressed by the presence of the condensate when the energy scale corresponding to the coherence length becomes of order the trimer binding energy in the absence of the condensate. We find that the Efimov scaling property is reflected in the critical values of the condensate coherence length at which the trimers are pushed into the continuum.
\end{abstract}

PACS. 03.65.Ge Solutions of wave equations: bound states - 03.75.Hh Static properties of condensates; thermodynamical, statistical, and structural properties - 67.85.-d Ultracold gases, trapped gases

\section{Introduction}

The study of few-body quantum mechanical bound states formed via strong short-range interactions has seen a tremendous boost after the observation of three-body states in cold atomic gases 1, 2. This is tied to a famous prediction by Vitaly Efimov that an infinitude of three-body bound states of identical bosons occurs at the point where the two-body subsystems have a bound state exactly at zero energy 3. These curious states have several interesting properties that are all in some way connected to the exponential factor $e^{\pi / s_{0}}$ where $s_{0}$ is called the scaling parameter. For instance, the three-body binding energies will scale with a factor of $e^{2 \pi / s_{0}} \sim 515$, becoming weaker by a huge factor for each subsequent state in the spectrum. Another key feature of these so-called Efimov states is that away from the resonance where the two-body binding energy vanishes, they display so-called borromean binding where no two-body subsystem is bound yet the three-body states remains bound (see Ref. 4 for a discussion of this phenomenon in the context of three or more particles).

The severe reduction in binding energy for subsequent states in the three-body spectrum strongly limits the number of states that one can expect to observe in experiments. However, for three-body systems with particles of different mass, this factor can be reduced considerably [5, 6] and more states could possibly be observed when using a mixture of different atomic species [2. Meanwhile, many ultracold atom experiments probe many-body systems which means that atomic few-body states should be considered as embedded in an environment. This has little influence on the few-body physics at very low density where theory and experiments match well, but the ability to push into higher density regimes is there and it is desirable in order to connect these studies to systems in other branches of physics such as condensed-matter and solid-state physics.

The question of what happens to few-body states when the Pauli principle influences one or several of the particles that make up the bound state has been discused in a number of recent papers $7,8,9,10,11,12$. Similarly, a number of works have addressed strongly interacting Bose gases at the border between the few-body and many-body perspective 13, 14, 15, 16, 17, 18. Also, some cases of one or more impurities in a condensate have been considered in the recent past $[19,20,21,22,23,24,25]$. In the current paper, we consider two heavy impurities that interact with a light bosonic particle which produces a small scale factor and thus should be a case where a number of three-body bound states may be observed. The effect of the manybody environment comes about since we assume that the light boson is part of a Bose condensate. This problem is reminiscient of electron-electron interactions mediated by phonons with the latter acting as the light bosonic particles that are condensed. However, we do take into account that the dispersion of the light boson is only linear at low momenta and then quadratic at higher momenta. The physics bears resemblence to a bosonic bath version of the Kondo problem studied in the condensed-matter community [26, 27, 28, 29, 30, 31]. 
The current study extends our recent paper 32 that suggests a direct and dramatic effect of the condensate environment on the Efimov three-body spectrum. Assuming that the condensate is in the weak coupling limit with a small and positive scattering length, $a_{B}>0$, we found in Ref. 32, that the condensate coherence length, $\xi=$ $1 / \sqrt{8 \pi n_{0} a_{B}}$ with $n_{0}$ the condensate gas density, may interfere with the three-body potential and reduce the binding until the states are eventually destroyed. Here we study this issue in a more quantative manner by numerical calculation of the spectrum as a function of $\xi$. This confirms and expands on the ideas presented in Ref. 32. Furthermore, we find that the spectral flow of the three-body binding energies with $\xi$ displays an Efimov scaling similar to what has been discussed for fermions recently [8, 9, 11. We also provide some appendices with a discussion of the technical details used here and in Ref. 32.

\section{Model}

Our basic setup consists of a quantum gas of bosons with mass $m$ and two embedded impurities with mass $M$ that we assume are much heavier than the bosons, i.e. $m \ll$ $M$ The interaction is of the density-density type and in second quantization the Hamiltonian becomes

$$
\begin{gathered}
H=\sum_{\boldsymbol{k}} \epsilon_{I}(\boldsymbol{k}) c_{\boldsymbol{k}}^{\dagger} c_{\boldsymbol{k}}+\sum_{\boldsymbol{k}} \epsilon_{B}(\boldsymbol{k}) b_{\boldsymbol{k}}^{\dagger} b_{\boldsymbol{k}} \\
+U_{B} \sum_{\boldsymbol{q}} n_{B}(\boldsymbol{q}) n_{B}(-\boldsymbol{q})+U_{I B} \sum_{\boldsymbol{q}} n_{B}(\boldsymbol{q}) n_{I}(-\boldsymbol{q}),
\end{gathered}
$$

where $c_{\boldsymbol{k}}$ are the impurity operators and $b_{\boldsymbol{k}}$ the boson operators. The interactions are assumed to be zero range with $n_{I}(\boldsymbol{q})=\sum_{\boldsymbol{k}} c_{\boldsymbol{k}+\boldsymbol{q}}^{\dagger} c_{\boldsymbol{k}}$ and $n_{B}(\boldsymbol{q})=\sum_{\boldsymbol{k}} b_{\boldsymbol{k}+\boldsymbol{q}}^{\dagger} b_{\boldsymbol{k}}$. The single-particle dispersions are $\epsilon_{I}(\boldsymbol{k})=\boldsymbol{k}^{2} / 2 M$ and $\epsilon_{B}(\boldsymbol{k})=$ $k^{2} / 2 m$ respectively. The impurity-boson coupling $U_{I B}$ will be addressed below. When $a_{B}$ is small we may use the weak coupling limit result $U_{B}=4 \pi \hbar^{2} a_{B} / m$. We use this expression throughout this paper and thus assume that the bosons are weakly interacting. In terms of the coherence length, $\xi$, this means that we are assuming that it is large. Below we will also push into the regime where $\xi$ becomes of the same order as the impurity-boson scattering length, $a$. In the regime where $\xi \leq|a|$ our method is not expected to hold quantitatively but should reveal the qualitative features of the setup.

In a weakly-interacting Bose gas, Bogoliubov theory applies to the light bosonic particles 33. We therefore transform to quasi-particles, $\gamma$ and $\gamma^{\dagger}$, via

$$
\begin{gathered}
b_{\boldsymbol{k}}^{\dagger}=u_{\boldsymbol{k}} \gamma_{\boldsymbol{k}}^{\dagger}+v_{\boldsymbol{k}} \gamma_{-\boldsymbol{k}} \\
b_{-\boldsymbol{k}}=v_{\boldsymbol{k}} \gamma_{\boldsymbol{k}}^{\dagger}+u_{\boldsymbol{k}} \gamma_{-\boldsymbol{k}},
\end{gathered}
$$

where $u, v$ are assumed real and symmetric in $\boldsymbol{k}$. The Hamiltonian is now

$$
\begin{gathered}
H=\sum_{\boldsymbol{k}} \epsilon_{I}(\boldsymbol{k}) c_{\boldsymbol{k}}^{\dagger} c_{\boldsymbol{k}}+\sum_{\boldsymbol{k} \neq 0} E_{B}(\boldsymbol{k}) \gamma_{\boldsymbol{k}}^{\dagger} \gamma_{\boldsymbol{k}} \\
+U_{I B} \sum_{\boldsymbol{q}} n_{B}(\boldsymbol{q}) n_{I}(-\boldsymbol{q})
\end{gathered}
$$

with $E_{B}(\boldsymbol{k})=\sqrt{U_{B} n_{0} \hbar^{2} \boldsymbol{k}^{2} / m_{B}+\left(\hbar^{2} \boldsymbol{k}^{2} / 2 m_{B}\right)^{2}}$ where $n_{0}$ is the condensate density. Constant terms have been dropped as they are are not relevant here. The boson density operator can now be expressed in terms of the quasiparticle operators. The $\boldsymbol{q}=0$ terms are

$$
n_{B}(\boldsymbol{q})=n_{0} \delta_{\boldsymbol{q} 0}+\sqrt{n_{0}}\left(b_{\boldsymbol{q}}^{\dagger}+b_{-\boldsymbol{q}}\right)+\sum_{\boldsymbol{k} \neq 0} b_{\boldsymbol{k}+\boldsymbol{q}}^{\dagger} b_{\boldsymbol{k}}
$$

Transforming to $\gamma$ operators one obtains

$$
\begin{gathered}
n_{B}(\boldsymbol{q})=n_{0} \delta_{\boldsymbol{q} 0}+\sqrt{n_{0}}\left(u_{\boldsymbol{q}}+v_{\boldsymbol{q}}\right)\left(\gamma_{\boldsymbol{q}}^{\dagger}+\gamma_{-\boldsymbol{q}}\right) \\
+\sum_{\boldsymbol{k} \neq 0}\left[u_{\boldsymbol{k}+\boldsymbol{q}} v_{\boldsymbol{k}} \gamma_{\boldsymbol{k}+\boldsymbol{q}}^{\dagger} \gamma_{-\boldsymbol{k}}^{\dagger}+u_{\boldsymbol{k}+\boldsymbol{q}} u_{\boldsymbol{k}} \gamma_{\boldsymbol{k}+\boldsymbol{q}}^{\dagger} \gamma_{\boldsymbol{k}}\right. \\
\left.+v_{\boldsymbol{k}+\boldsymbol{q}} v_{\boldsymbol{k}} \gamma_{-\boldsymbol{k}-\boldsymbol{q}} \gamma_{-\boldsymbol{k}}^{\dagger}+v_{\boldsymbol{k}+\boldsymbol{q}} u_{\boldsymbol{k}} \gamma_{-\boldsymbol{k}-\boldsymbol{q}} \gamma_{\boldsymbol{k}}\right] .
\end{gathered}
$$

The number of terms is reducible by using $u \rightarrow 1$ and $v \rightarrow$ 0 in the weakly interacting limit. This eliminates the $\gamma \gamma$ and $\gamma^{\dagger} \gamma^{\dagger}$ pieces. Also, the first term will yield a constant proportional to the impurity density when inserted in the Hamiltonian so we drop this also. The most problematic term is the one linear in $\gamma$ and $\gamma^{\dagger}$ with a prefactor of $\sqrt{n_{0}}$. Here we will assume that the condensate density is small, which in the weak-coupling limit, is the same as assuming that $n_{B}$ is small. The impurity-boson interaction term now reduces to

$$
U_{I B} \sum_{\boldsymbol{q} \boldsymbol{k} \boldsymbol{k}^{\prime}} c_{\boldsymbol{k}-\boldsymbol{q}}^{\dagger} c_{\boldsymbol{k}} \gamma_{\boldsymbol{k}^{\prime}+\boldsymbol{q}}^{\dagger} \gamma_{\boldsymbol{k}^{\prime}},
$$

which is a standard interaction term for a zero-range interaction of strength $U_{I B}$. The effective Hamiltonian is then

$$
\begin{aligned}
H= & \sum_{\boldsymbol{k}} \epsilon_{I}(\boldsymbol{k}) c_{\boldsymbol{k}}^{\dagger} c_{\boldsymbol{k}}+\sum_{\boldsymbol{k} \neq 0} E(\boldsymbol{k}) \gamma_{\boldsymbol{k}}^{\dagger} \gamma_{\boldsymbol{k}} \\
& +U_{I B} \sum_{\boldsymbol{q} \boldsymbol{k} \boldsymbol{k}^{\prime}} c_{\boldsymbol{k}-\boldsymbol{q}}^{\dagger} c_{\boldsymbol{k}} \gamma_{\boldsymbol{k}^{\prime}+\boldsymbol{q}}^{\dagger} \gamma_{\boldsymbol{k}^{\prime}}
\end{aligned}
$$

which corresponds to impurity particles with dispersion $\epsilon_{I}(\boldsymbol{k})$ interacting with Bose gas particles with dispersion $E(\boldsymbol{k})$ through a contact interaction with strength $U_{I B}$. In the case where $E(\boldsymbol{k})$ is linear in $\boldsymbol{k}$, this corresponds to a system of (heavy) electrons interacting with phonons through a non-dispersive zero-range intearction.

Before we proceed to solve the three-body problem of two heavy impurities and one light boson in a condensate using this effective Hamiltonian, a couple of remarks are in order. The states we discuss here are not the absolute ground state of the system, but rather few-body resonances similar to the ones that can be studied in cold atomic gases 2. The true ground state in the current model is more likely a bound state of both impurities and all the bosons in the condensate. However, such large cluster are not formed on the typical experimental timescale in cold atomic gases. An alternative bound state to consider would be one with two light bosons and one impurity. This type of system has a very large Efimov scale factor $e^{\pi / s_{0}}$ [5, 6] and observation of Efimov states is therefore not favoured. In constrast, two heavy impurities and one light particle has a small scale factor and the spectrum of Efimov states is expected to be dense (see Appendix C for a discussion of this feature using the Born-Oppenheimer approximation). 


\subsection{Born-Oppenheimer Approximation}

Since we model the impurity-boson interaction by a zerorange two-body potential, we have

$$
V(\boldsymbol{r})=V_{0}[\delta(\boldsymbol{r}-\boldsymbol{R} / 2)+\delta(\boldsymbol{r}+\boldsymbol{R} / 2)],
$$

where the impurities are located at $\pm \boldsymbol{R} / 2$. Regularization is necessary and we discuss this momentarily. We will now use the Born-Oppenhimer approximation. Here the light particle motion is solved while assuming that $\boldsymbol{R}$ is unaltered. This is done for all values of $\boldsymbol{R}$, and in turn one obtains an effective Schrödinger equation for the heavy particles which depends on $\boldsymbol{R}$. This implies that we consider $\boldsymbol{R}$ an adiabatic variable that changes much slower than the position of the light particle.

The Schrödinger equation for the light particle of mass $m$ in the potential of Eq. (11) is

$$
H \phi=E_{R} \phi,
$$

where $E_{R}$ is the energy and $\phi$ the wave function. Delta functions are conveniently handled in momentum space and we thus transform our equation into

$$
E(\boldsymbol{k}) \phi(\boldsymbol{k})+\frac{1}{(2 \pi)^{3}} \int d^{3} k^{\prime} \phi\left(\boldsymbol{k}^{\prime}\right) V\left(\boldsymbol{k}-\boldsymbol{k}^{\prime}\right)=E_{R} \phi(\boldsymbol{k}) .
$$

For the case at hand we have $V(\boldsymbol{q})=2 V_{0} \cos \left(\boldsymbol{q} \cdot \frac{\boldsymbol{R}}{2}\right)$ and in turn

$$
\phi(\boldsymbol{k})=\frac{-2 V_{0}}{(2 \pi)^{3}} \frac{1}{E(\boldsymbol{k})-E_{R}} \int d^{3} k^{\prime} \cos \left(\frac{\left(\boldsymbol{k}-\boldsymbol{k}^{\prime}\right) \cdot \boldsymbol{R}}{2}\right) \phi\left(\boldsymbol{k}^{\prime}\right) .
$$

One may now multiply by $\cos (\boldsymbol{k} \cdot \boldsymbol{R} / 2)$ and integrate both sides with respect to $\boldsymbol{k}$. Using the assumptions that $\phi$ is even in $\boldsymbol{k}$ (solutions with $s$-wave symmetry), a couple of trigonometric manipulations leads us to the equation

$1=-\frac{V_{0}}{(2 \pi)^{3}} \int d^{3} k \frac{1}{E(\boldsymbol{k})-E_{R}}-\frac{V_{0}}{(2 \pi)^{3}} \int d^{3} k \frac{\cos (\boldsymbol{k} \cdot \boldsymbol{R})}{E(\boldsymbol{k})-E_{R}}$.

It is now necessary to face the issue of regularization of the interaction, which implies that the parameter $V_{0}$ be substituted for the physical impurity-boson scattering length, $a$, while at the same time absorbing the divergence that appears in the first integral on the right-hand side of Eq. (15). This can be done elegantly using pseudopotentials [34 35], although here we will use the more traditional Lippmann-Schwinger equation approach. The scattering equation for the impurity-boson problem with a zero-range two-body interaction is

$$
\frac{1}{V_{0}}=\frac{\mu}{2 \pi a \hbar^{2}}-\frac{1}{(2 \pi)^{3}} \int d^{3} k \frac{1}{\epsilon_{\boldsymbol{k}}^{\mu}}
$$

with the reduced mass $\mu=m M /(m+M)$ and the reduced energy $\epsilon_{k}^{\mu}=\hbar^{2} k^{2} / 2 \mu$. Since we work in the limit $m \ll M$, we take $\epsilon_{\boldsymbol{k}}^{\mu}=\epsilon_{\boldsymbol{k}}$ and $\mu=m$. Here we use the bare singleparticle dispersion, $\hbar^{2} \boldsymbol{k}^{2} / 2 m$, for the bosons and not $E(\boldsymbol{k})$.
We do so because the impurity-boson scattering length, $a$, is defined in vacuum and the scattering equation must thus be the vacuum version as well. Combining Eq. (16) and Eq. (15) we obtain the regularized momentum space Schrödinger equation

$$
\begin{aligned}
& \frac{m}{2 \pi a \hbar^{2}}=-\frac{1}{(2 \pi)^{3}} \int d^{3} k\left[\frac{1}{E(\boldsymbol{k})-E_{R}}-\frac{1}{\hbar^{2} \boldsymbol{k}^{2} / 2 m}\right] \\
& -\frac{1}{(2 \pi)^{3}} \int d^{3} k \frac{\cos (\boldsymbol{k} \cdot \boldsymbol{R})}{E(\boldsymbol{k})-E_{R}},
\end{aligned}
$$

which can be written

$$
\begin{aligned}
& \frac{R}{a}=-\frac{2}{\pi} \alpha R \int_{0}^{\infty} d x\left[\frac{x^{2}}{\left[x^{4}+A^{2} x^{2}\right]^{1 / 2}+1}-1\right] \\
& -\frac{2}{\pi} \int_{0}^{\infty} d x \frac{x \sin (\alpha R x)}{\left[x^{4}+A^{2} x^{2}\right]^{1 / 2}+1}
\end{aligned}
$$

when we define the dimensionless quantities $\alpha^{2}=-2 m E_{R} / \hbar^{2}$ and $A=1 /(\alpha \xi)$. The two integrals in Eq. (18) are discussed in various limits in Appendix A

For non-interacting bosons with $a_{B}=0$, we have $\xi=$ $\infty$ and correspondingly $A=0$. Using the analytical results in Appendix $\mathrm{A}$, one obtains the formula

$$
\alpha R=\frac{R}{a}+e^{-\alpha R}
$$

When the impurity-boson interaction is on resonance, $|a|=$ $\infty$, the solution is $\alpha R=x_{0} \sim 0.567$. One can obtain the solution as a power series in the variable $R / a$, and to lowest order one finds

$$
E_{R}=-\frac{\hbar^{2} x_{0}^{2}}{2 m R^{2}}\left[1+\frac{1}{x_{0}^{2}\left(1+e^{x_{0}}\right)} \frac{R}{a}\right] .
$$

In the limit $R \gg a$, the second term on the right-hand side of Eq. (19) goes to zero and one obtains the well-known result

$$
E_{R}=-\frac{\hbar^{2}}{2 m a^{2}} .
$$

The physical interpretation is that the light particle becomes bound to either of the impurities which are effectively just static potentials in space. The binding energy is the one appropriate for a zero-range two-body potential and the solution only exists for $a>0$ where the impurityboson system can bind.

\section{Three-Body Bound States}

The two-body bound state solutions of a single impurity and a light bosonic particle were discussed in details in Ref. 32 and we will not repeat this discussion here but rather move onto the three-body problem. The influence of the condensate on the light particle goes through the dispersion, $E(\boldsymbol{k})$, and then into the properties of the heavy-heavy-light three-body bound states via the BornOppenheimer approximation. In turn, we need to determine the impurity-impurity potential, $E_{R}$, which is given 


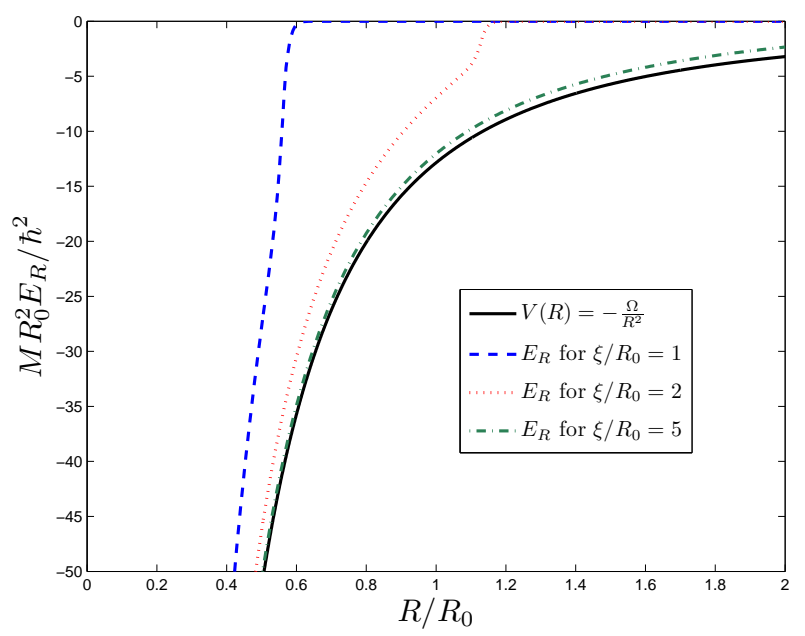

Fig. 1. Potential, $E_{R}$, between the two impurities in the BornOppenheimer approximation as function of $R / R_{0}$ where $R_{0}$ is the short-distance cut-off (see discussion in text) which is the unit of length. $E_{R}$ is shown for $\xi / R_{0}=1$ (dashed line), $\xi / R_{0}=$ 2 (dotted line), and $\xi / R_{0}=5$ (dot-dashed line). For comparison, the solid line shows the potential, $V(R)=-\Omega / R^{2}$, when the light particle is not part of a condensate. In all cases shown, the strength of the potential is $\Omega=40 x_{0}^{2}$ corresponding to a mass ratio $M / m=80$.

implicitly through $\alpha$ which is obtained by solving Eq. (18). The first integral in Eq. (18) is exactly solvable, see Appendix A. However, the second term in Eq. (18) is more difficult both analytically and numerically. As discussed in Ref. 32, it can be solved using different approximate approaches that are discussed in more detail in Appendix The main message is that the presence of a condensate influencing the light particle tends to suppress the attractive $1 / R^{2}$ potential that is obtained in the non-condensed limit where $\xi \rightarrow \infty$ and $E_{R} \propto-1 / R^{2}$ (see the solid line in Fig. 10.

\subsection{Impurity-impurity Potential}

In the non-condensed limit $\xi \rightarrow \infty$, a finite value of $a$ would imply that the $-1 / R^{2}$ behaviour is cut off at $R \sim$ $|a|$ [5, 6]. The qualitative implication is that whichever is smaller of $|a|$ and $\xi$ will cause $E_{R}$ to fall off to zero faster than $-1 / R^{2}$ for $R \gtrsim \min (|a|, \xi)$. Here we will focus on the limit where the impurity-light boson two-body interaction is resonant, i.e. we assume that $a \rightarrow \infty$ and thus we neglect the left-hand side of Eq. 18. The modification of the $-1 / R^{2}$ behaviour is therefore controlled by the value of $\xi$.

As discussed in Ref. [32, the numerical solution is increasingly difficult to find for $R / \xi \rightarrow x_{0}$, but strongly indicates that $\alpha R$ goes to zero at a finite value of $R / \xi$. The behaviour can be accurately fitted by the function

$$
\alpha R=\frac{x_{0}}{2}+\frac{x_{0}}{2}\left[1-\left(\frac{R}{x_{0} \xi}\right)^{2}\right]^{1 / 2}
$$

for $\alpha R>x_{0} / 2$. Notice that there is a typo in 32 where the first term $x_{0} / 2$ is missing and a factor of $1 / 2$ is missing from the second term. Alternatively, we can approach the limit of small $R \sim \xi$ and $\alpha \xi$ by considering the limit $A^{2} \gg 1$ before doing the integrations in Eq. (18). The asymptotic expressions are discussed in Appendix $\mathrm{A}$ and given in Eqs. (30) and (38). In this limit, Eq. (18) yields

$$
(\alpha \xi)^{2}=\frac{2}{\pi} \frac{\frac{\xi}{R}-\frac{R}{\xi}}{\frac{R}{\xi}-1}<0 .
$$

This is manifestly negative and implies that $E_{R}$ changes sign as $R \sim \xi$. We caution that in the limit $R \rightarrow \xi$ in Eq. (23) the result is $-\frac{4}{\pi}$. In turn $A^{2}$ is of order 1 which is conflicting with the limit we used to derive the expression. In any case, it strongly suggests that $E_{R}$ goes to zero at a finite $R$, and we will assume that this is generally the case for any finite value of $\xi$.

Combining the accurate fit in Eq. (22) with the fact that $E_{R}$ must vanish beyond some finite $R$, we now introduce our model to describe the impurity-impurity potential. We will assume that the fit in Eq. (22) holds from $R=0$ to $R / \xi=x_{0}$, i.e. when the expression under the root in Eq. (22) is non-negative. As discussed in Ref. 32], the presence of the condensate influences the potential $E_{R}$ at large distance by making it approach zero rapidly for $R \sim \xi x_{0}$. Furthermore, we assume that as long as $E_{R}$ goes to zero faster than $1 / R^{2}$ when $R \sim \xi x_{0}$, the functional form is not important. We therefore use a FermiDirac function that changes rapidly from 1 to 0 around $R / \xi=x_{0}$. In total, the potential, $E_{R}$, in the spherically symmetric $s$-wave channel between the two impurities becomes

$$
E_{R}=-\frac{\hbar^{2}}{2 m R^{2}} \frac{x_{0}^{2}}{4}\left[1+\sqrt{1-\left(R / x_{0} \xi\right)^{2}}\right]^{2} F\left[R, \xi x_{0}, w\right],
$$

where the Fermi-Dirac function is

$$
F[x, y, z]=\frac{1}{1+e^{(x-y) / z}}
$$

and $w$ is a width parameter that we set to $w=0.01 R_{0}$. Here $R_{0}$ is a short-distance cut-off that we discuss momentarily. We have checked that small changes in $w$ does not affect the physics discussed in this paper. In the limits where $\xi \rightarrow \infty$, we recover the attractive $1 / R^{2}$ potential that gives rise to the Efimov effect (see Appendix C). The resulting potentials for different values of $\xi$ are shown in Fig. 1 for mass ratio $M / m=80$. We notice in the plot that already for $\xi / R_{0}=5$, the limiting $-1 / R^{2}$ behaviour is approached.

The number of three-body Efimov state expected in the absence of the condensate $(\xi=\infty)$ is

$$
N_{B} \approx \frac{s_{0}}{\pi} \log \left[\frac{|a|}{R_{0}}\right]
$$

where $R_{0}$ is a short-distance cut-off which we return to below (also discussed in Appendix C). For finite $\xi$ we clearly see the attractive region shrinking in Fig. 1 and one can 


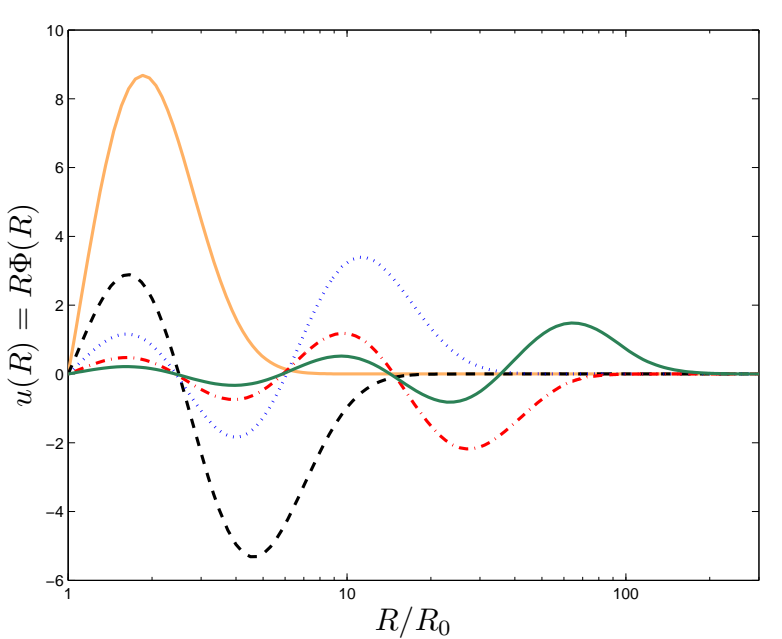

Fig. 2. Eigenstates, $u(R)$, of the Schrödinger equation in Eq. (27) for $M / m=80$ and with a short-distance cut-off at $R=R_{0}$ as function of $R / R_{0}$. The horizontal axis has a logarithmic scale for increased visibility. Notice how the first node of the wave function coincides for the four excited states, the second node for the three highest excited states, and the third node for the two highest excited excited states. This is a key feature of three-body Efimov states.

in turn replace $a$ by $\xi$ in the formula for $N_{B}$ as a crude estimate. In general, whichever is smaller of $a$ and $\xi$ sets the scale for the extend of the attraction and thus the number of three-body Efimov states that can be accomodated. This is the basic physical idea presented in Ref. 32 . In the next section we proceed to discuss this idea quantitatively using the potential in Eq. (24).

As noted in Ref. 32, the current model does not imply any influence of the condensate on the short distance behaviour of the impurity-impurity potential. Short distance corresponds to high momentum for which the singleparticle dispersion of the light bosonic particles, $E(\boldsymbol{k})$, goes to the free particle dispersion. The short distance cut-off, $R_{0}$, introduced above should therefore be interpreted and treated in the usual manner. While it is often considered merely an effective parameter (denoted the three-body parameter), in atomic systems it appears to have a much more direct physical connection to the twobody inter-atomic potentials and can be related to the van der Waals length, $r_{\mathrm{vdW}}$ 36, 37, 38, 39, 40, 41, 42, 43, 44, 45. In the current study we assume $m \ll M$. In this case it is quite natural from the discussion above that $E_{R}$ will be regularized at short distance by a cut-off that comes from the impurity-impurity potential. In other words, the regularization is given by the short-distance behaviour of the two-body potential between the two impurities.

\subsection{Solutions and Spectral Flow}

We now present a set of solutions for the wave functions, $\Phi(R)$, and the energies, $E$, of the Schrödinger equation in the spherically symmetric $s$-wave channel for the potential
$E_{R}$ in Eq. (24), i.e.

$$
\left[-\frac{\hbar^{2}}{M} \frac{d^{2}}{d R^{2}}+E_{R}\right] u(R)=E u(R),
$$

where $u(R)=R \Phi(R)$. We take a large $M / m=80$ when computing the scale factor, $s_{0}$. The large ratio of $M / m$ is employed to have several states to study as a function of $\xi$. For other mass ratios we find similar behavior. The scale factor can now be computed using the formulas in Appendix C and we obtain $s_{0}=3.55$ or $e^{\pi / s_{0}}=2.42$. We also need to specify the short distance cut-off and here we take $R_{0}$. Since we assume that $|a| \rightarrow \infty$, the limit where also $\xi \rightarrow \infty$ in principle has an infinite number of threebody bound states due to the Efimov effect. The numerical precision of course limits the number that can actually be found in a given calculation and we find $N_{B}=8$ for large $\xi$ as we discuss below.

In Fig. 2 we show $u(R)$ for the five lowest bound states obtained by numerical solution of the Schrödinger equation for large $\xi$. This was done by direct integration of the inner part of the potential and then a comparison to the exact bound state exponential tail outside the potential (with the boundary defined so that the potential can be neglected, i.e. for $R>\xi x_{0}$, see Fig. 1). The five bound states in Fig. 2 nicely follow the rules of adding an additional node to the wave function as one moves up in the spectrum. Notice the first zero of the four excited states which is at the same value of $R / R_{0}$ (and likewise for the second node of the three highest states, and third node for the two highest states). This is a clear manifestation of the scale invariance of the wave function that is the essence of the Efimov effect (see Appendix C). Armed with the knowledge that our numerical procedure produces an Efimov spectrum with a number of states, we proceed to consider finite values of $\xi$.

The modifications to the wave functions are illustrated in Fig. 3 for the fourth excited state (left panel) and third excited state (right panel). In both cases we see that for finite $\xi / R_{0}$, the wave function is mainly modified at large distance. In fact, the wave function amplitude seems to spread to large distance in comparison to the $\xi / R_{0}=\infty$ case. This observation is perfectly consistent with the discussion above and the subsequent expectation that the presence of a condensate affecting the light particle should influence the large distance bahaviour. This flow of probability to the outer regions can be understood from the potential form in Eq. (24) or more precisely from Eq. (22). The potential goes rapidly to zero at $R / \xi \sim x_{0}$ as shown in Fig. 1 and the strength of the attractive $1 / R^{2}$ decreases with decreasing $\xi$. This means that the wave function can more easily leak out into the region beyond $R / \xi=x_{0}$ as we see in Fig. 3. An increasing amplitude in the region of space where the potential is negligible implies that the binding energy must subsequently decrease since the potential is purely attractive. We will now quantify this feature and demonstrate the spectral flow with $\xi$ and its connection to Efimov scaling.

The variation of the binding energy is shown in Fig. 4 as a function of $\xi / R_{0}$. The plot has been rescaled on the 

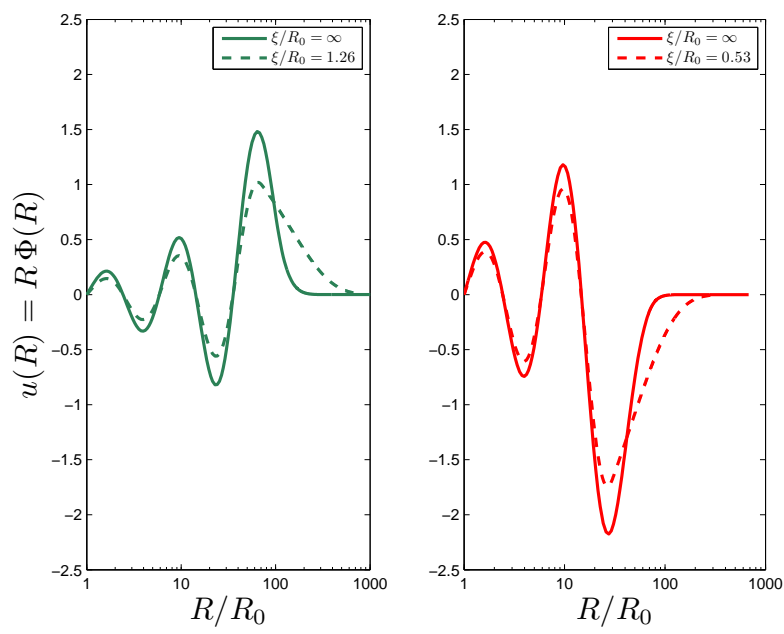

Fig. 3. Eigenstates, $u(R)$, as in Fig. 2 but for both $\xi / R_{0}=\infty$ and for finite $\xi$. Left panel: The fourth excited state for $\xi / R_{0}=$ $\infty$ (solid line) and $\xi / R_{0}=1.26$ (dashed line). Right panel: The third excited state for $\xi / R_{0}=\infty$ (solid line) and $\xi / R_{0}=0.53$ (dashed line). Notice the transfer of wave function amplitude to larger $R / R_{0}$ when $\xi$ is finite. This shows the modifications caused by the condensate at large distance.

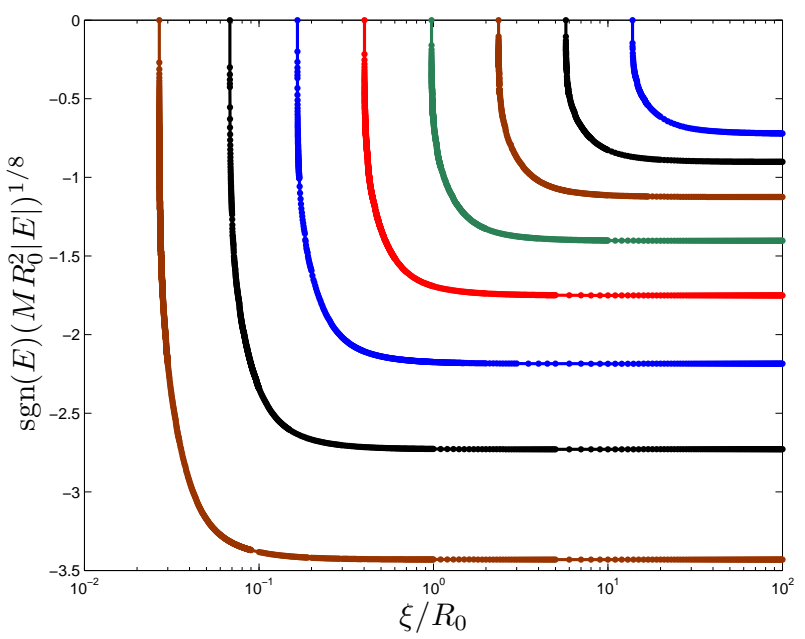

Fig. 4. Spectral flow as a function of $\xi / R_{0}$ of the energy for the four excited states shown in Fig. 2 The vertical axis has been scale by the power $1 / 8$, while the horizontal axis is logarithmic. This has been done to make the connection to the Efimov scaling factor, $e^{\pi / s_{0}}$, clearly visible in the plot. The turning points at which the binding energy starts to decrease rapidly is roughly at the point where $\hbar^{2} / M \xi^{2}$ is equal to the binding energy of the state for $\xi=\infty$.

vertical axis by a power of $1 / 8$ (see axis label), while the horizontal axis is logarithmic. This helps visualize the presence of Efimov scaling as $\xi$ changes. Indeed in the plot we clearly see the self-similar behaviour of the flow. The weakest bound state is influenced by the condensate at the largest $\xi$, and subsequently has the largest critical value, $\xi_{c}$, at which its binding goes to zero. Then the next deeper bound state repeats the same flow as $\xi$ decreases still further and so on. The jump in the energy seen in

\begin{tabular}{|c|c|c|c|c|}
\hline$n$ & 1 & 2 & 3 & 4 \\
\hline$E_{0}^{n-1} / E_{0}^{n}$ & 6.23 & 5.92 & 5.88 & 5.89 \\
$\xi_{c}^{n-1} / \xi_{c}^{n}$ & 2.54 & 2.43 & 2.42 & 2.42 \\
\hline$n$ & 5 & 6 & 7 & $\infty$ \\
\hline$E_{0}^{n-1} / E_{0}^{n}$ & 5.85 & 5.89 & 6.01 & 5.87 \\
$\xi_{c}^{n-1} / \xi_{c}^{n}$ & 2.42 & 2.43 & 2.41 & 2.42 \\
\hline
\end{tabular}

Table 1. Ratios of energies and of critical coherence lengths, $\xi_{c}$, at which the binding energy of the states vanish. The index $n$ runs from 0 (ground states) to 7 (seventh excited states), while the column with $n=\infty$ gives the analytical value obtained for $\xi=\infty$ and on resonance where $a=\infty$. The latter is $e^{2 \pi / s_{0}}$ for the energy ratios and $e^{\pi / s_{0}}$ for the coherence length ratios.

Fig. 4 happens when the energy of the three-body state in the limit $\xi / R_{0}=\infty$ (absence of condensate effects) is roughly equal to $E_{\xi}=\hbar^{2} / M \xi^{2}$. This means that we have a sort of 'window of opportunity' for a given $\xi$ value within which modifications to three-body states can be expected. By comparison to the spectral flow of Efimov three-body states in the presence of a Fermi sea [8, 9, we see that the Fermi energy scale, $E_{F}$, is replaced by $E_{\xi}$. Apart from this, the two flows of the three-body binding energies are very similar. Note, however, that this mapping of similarities does not hold for the two-body impurity-boson system as discussed in Ref. 32 where light bosons can instead be mapped to a problem with heavy fermions (at least within the Born-Oppenheimer approach).

The signatures of Efimov scaling are clearly seen in Fig. 4. To further quantify this finding we provide an overview of the different scaling factors of adjacent states in Tab. 1. The table contains ratios of binding energies and also ratios of critical coherence lengths, $\xi_{c}$, where the binding energies vanish. The scaling factors obtained numerically from the binding energies are close to the analytically calculated ratios and only for the lowest and highest states, do we see deviations. This is not unexpected. In the case of the lowest state, the binding energy is very large and the short-distance part of the potential plays a role through the cut-off $R_{0}$. For the highest excited state that has substantial amplitude at larger distances, the deviation comes from sensitivity to the fact that the potential is modified from the attractive inverse square form at large distance but nevertheless this remains a small effect. The story is similar for the ratio of critical coherence lengths as seen in Tab. 1 .

\section{Discussion}

Using the Born-Oppenheimer approximation, we have studied both qualitatively and quantitatively, the three-body bound states of two heavy impurities in a Bose condensate of light bosonic particles where the impurity-boson interaction is close to resonance. This correspond to large impurity-boson scattering length and opens up the study of Efimov three-body states and their scaling properties in the presence of a background medium of condensed bosons. A previous study 32 argued that this implies a 
competition between the impurity-boson scattering length and the condensate coherence length, and that the smallest of those scales will determine the estimated number of Efimov three-body states to expect. In fact, when the impurity-boson scattering length diverges, the condensate coherence length is the sole scale left to determine this number and it becomes an effective large distance cut-off on the attractive inverse-square potential that is the origin of the Efimov effect.

We solved the bound state problem of the two impurities interacting via the Born-Oppenheimer potential obtained by integrating out the light bosonic particle that was assumed to have a non-trivial dispersion due to the presence of the condensate (with a linear momentum dependence at small momenta). This allowed us to study the flow of the bound state energies with condensate coherence length. We find that once the energy scale given by the coherence length becomes of order the three-body binding energy without any condensate, then the three-body state becomes strongly modified and eventually gets pushed into the continuum. This is similar to the behaviour seen for Efimov states in the presence of a degenerate Fermi sea [8, 9. In fact, one can map the behaviours by changing Fermi energy, $E_{F}$, to the coherence length energy scale, $E_{\xi}=\hbar^{2} / M \xi^{2}$, where $\xi$ is the coherence length. However, one needs to notice that $M$ is the impurity mass. While we have assumed that $M$ is much larger than the mass of the degenerate bosons, we expect that a similar kind of scaling can be found for other mass ratios. This would require a formalism more general than the Born-Oppenheimer approach used here and in Ref. 88. Furthermore, it would be interesting to study the influence of fluctuations in the Bose condensate in similar fashion to what was discussed in Ref. 8] for a Fermi sea where fluctuations in the form of particle-hole pairs have been considered.

In order to experimentally address the physics discussed here, a mass-imbalanced system is needed. As noted above, we do expect similar behaviour also for mass-balanced cases but this is not the topic of the present discussion. To achieve large mass ratios some recent experiments with Helium are of interest [46, 47] and in particular the possibility to pursue mixtures of ${ }^{4} \mathrm{He}$ and ${ }^{87} \mathrm{Rb}$ [48]. Other mixtures of interest are ${ }^{7} \mathrm{Li}$ and ${ }^{133} \mathrm{Cs}$ 49, ${ }^{7} \mathrm{Li}$ and ${ }^{87} \mathrm{Rb}$ [50], or mixtures of Ytterbium isotopes (mass numbers 168-176) [51,52 with ${ }^{7} \mathrm{Li}$ or ${ }^{4} \mathrm{He}$. Estimates of the effects on the number of bound states the two former cases have been given in Ref. 32. For smaller mass imbalances we mention ${ }^{23} \mathrm{Na}$ with ${ }^{40} \mathrm{~K}$ [53] and ${ }^{87} \mathrm{Rb}$ with ${ }^{133} \mathrm{Cs}$ [54,55, 56] as promising experimentally studied systems. This requires theoretical ventures beyond the Born-Oppenheimer approximation which we will pursue in the future. A key issue is experimental control over both the condensate coherence length, $\xi$, and the impurity-boson scattering length, $a$. The most common way to tune such parameters is through magnetically tunable Feshbach resonances but also optical Feshbach resonances have been studied [57. One could then imagine an optical tuning of one of these parameters and a magnetic tuning of the other. Another possibility is to use the fact that $\xi=1 / \sqrt{8 \pi n_{0} a_{B}}$ depend on not only the boson-boson scattering length, $a_{B}$, but also on the condensate density, $n_{0}$. Changing $n_{0}$ could therefore accomplish similar things.

There is great experimental interest in strongly interactin Bose gases at the moment both in three [58,59, 60, 61, 62 and in two dimensional systems 63 64, 65. Recent theoretical works addressing such experiments [66, 67. have shown the importance of the few-body states (twoand three-body) present in the strongly interacting gases. In similar experiments with mass-imbalanced mixtures of bosons, the few-body states should play an equally important role. As we have discussed, the number of bound states to be expected depends on the intra-species interaction strength and the results presented here can guide one in estimating the bound state spectrum.

The main feature of our setup is the linear dispersion at low energy of the bosons. We obtain this through the presence of a condensate background medium. However, it is easy to imagine other ways to achieve the same thing. Systems like Graphene 68 and topological insulator surfaces 69,70] are examples of linear dispersions at low energy. The influence of the electrons in these systems on impurities is a topic of great interest in the solid-state and condensed-matter communities 71. More generally, we may ask what happens with modified dispersion and three-body systems, and in different dimensionalities. For instance, applying spin-orbit coupling to atomic systems 72. has recently been shown to provide an interesting Efimov spectrum due to a modification of the dispersion [73]. The recently discovered Efimov effect for spin-polarized fermions in two dimensions 74 and the presence of deeply bound borromean states [75] implies that such a system could have a transition of its Fermi surface and thus of the dispersion. These are very interesting cases in which to study few-body physics in a many-body background.

\section{A Integrals}

The first integral that appears in Eq. (18) can be solved exactly and yields

$$
\begin{aligned}
& \int_{0}^{\infty} d x\left[\frac{x^{2}}{\left[x^{4}+x^{2} /(\alpha \xi)^{2}\right]^{1 / 2}+1}-1\right]=-\frac{2}{\pi} \frac{1}{\alpha \xi}[-1 \\
& +\frac{(\alpha \xi)^{2}}{\sqrt{2+8(\alpha \xi)^{4}}} \sqrt{\sqrt{1+4(\alpha \xi)^{4}}+1} \\
& \times\left(\operatorname{ArcTan}\left[\sqrt{\frac{2}{\sqrt{1+4(\alpha \xi)^{4}}-1}}\right]-\pi\right) \\
& +\frac{(\alpha \xi)^{2}}{\sqrt{2+8(\alpha \xi)^{4}}} \sqrt{\sqrt{1+4(\alpha \xi)^{4}}-1} \\
& \times \operatorname{ArcTanh}\left[\sqrt{\frac{2}{\sqrt{1+4(\alpha \xi)^{4}}+1}}\right]
\end{aligned}
$$


To first order in $A^{2}=\frac{1}{(\alpha \xi)^{2}}$ we obtain the simple expression

$$
-\frac{2}{\pi}\left(1+\frac{1}{4} A^{2}\right)
$$

In the opposite limit where $A^{2} \gg 1$, the integral becomes

$$
-A-\frac{\pi}{2} \frac{1}{A}
$$

to leading order in $A$.

The second integral

$$
\int_{0}^{\infty} d x \frac{x \sin (\alpha R x)}{\left[x^{4}+A^{2} x^{2}\right]^{1 / 2}+1}
$$

is significantly more complicated due to the oscillatory factor. Numerically it is one of the most difficult tasks to compute highly oscillatory functions. Fortunately, there are limits in which analytical results can still be obtained. This is the case for the important limit of $A$ small. To second order in $A^{2}$ we have

$$
\begin{gathered}
\int_{0}^{\infty} d x \frac{x \sin (\alpha R x)}{x^{4}+\left(1+A^{2} / 2\right) x^{2}-A^{4} / 8}= \\
\frac{\pi}{2 H}\left[F^{2} \exp (-\alpha R F)-G^{2} \cos (\alpha R G)\right],
\end{gathered}
$$

where

$$
F=\frac{2+A^{2}+\sqrt{4+4 A^{2}+3 A^{4}}}{4}, \quad G=F-1-\frac{A^{2}}{2}
$$

and $H=F^{2}+G^{2}$. To second order this reduces to the simple result

$$
\frac{\pi}{2} \exp \left(-\alpha R-\frac{1}{4} \alpha R A^{2}\right)
$$

Using the results of Eqs. 29] and 34 in Eq. (18) we obtain the eigenvalue equation for $\alpha$

$$
\alpha R+\frac{1}{4} \frac{R}{\alpha \xi^{2}}=\frac{R}{a}+\exp \left(-\alpha R-\frac{1}{4} \frac{R}{\alpha \xi^{2}}\right),
$$

which is our result for general scattering lengths. Solutions must still be consistent with the approximation $A^{2} \ll 1$ that was used to arrive at this result. We have checked this a posteriori and find that a sufficient condition is $R \ll \xi$. However, this is not a necessary condition as can be seen in the following way. Consider the limit of large $R$ in Eq. (35) defined as the limit in which we drop the exponential term and find that Eq. (35) reduces to

$$
\frac{1}{a}=\alpha+\frac{1}{4 \alpha \xi^{2}},
$$

We thus see that the two-body problem is recovered in this limit. Note that this result is consistent with the approximation $A^{2} \ll 1$ for large $\xi$ (which is a basic assumption on the formalism as discussed in Sec. 21).
The regime of $A^{2} \gg 1$ is more difficult. We may consider the extreme limit where we approximate the integrand in Eq. (31) to obtain

$$
\begin{aligned}
& \int_{0}^{\infty} d x \frac{x \sin (\alpha R x)}{A x+1}= \\
& \frac{\frac{2 A}{\alpha R}-2 \operatorname{Ci}\left[\frac{\alpha R}{A}\right] \sin \left(\frac{\alpha R}{A}\right)-\cos \left(\frac{\alpha R}{A}\right)\left(2 \operatorname{Si}\left[\frac{\alpha R}{A}\right]-\pi\right)}{\sqrt{2 \pi} A^{2}}
\end{aligned}
$$

which is the sine transform of the function $x /(A x+1)$. Here $\operatorname{Si}(x)$ and $\mathrm{Ci}(x)$ are the Sine and Cosine integrals. To be more precise one should split the integral in Eq. (31) according to values of $x^{2}<A^{2}$ and $x^{2}>A^{2}$. However, the presence of oscillatory factor $\sin (\alpha R x)$ implies that the latter part will give a very small contribution for $A^{2} \gg 1$ and we neglect it. The argument of the above expansion is $\alpha R / A=\frac{R}{\xi} \frac{1}{(\alpha \xi)^{2}}$. If we assume that $R \sim \xi$, this is a large number $\left(A^{2} \gg 1\right)$ and we can expand the result above to obtain

$$
\int_{0}^{\infty} d x \frac{x \sin (\alpha R x)}{A x+1} \rightarrow \frac{\xi}{R}-\frac{1}{2} \pi^{2}(\alpha \xi)^{2}
$$

to second order in $\alpha \xi$ which is assumed small.

\section{B Impurity Potential at Unitarity}

We now assume that $a$ diverges and thus neglect the lefthand side of Eq. (18). Furthermore, we assume that $A^{2}=$ $\frac{1}{(\alpha \xi)^{2}} \ll 1$ and thus truncate the integrals. Eq. (18) now reduces to the simple form

$$
\alpha R+\frac{1}{4} \alpha R A^{2}=\exp \left(-\alpha R-\frac{1}{4} \alpha R A^{2}\right) .
$$

This is readily solved since we have $x_{0}=\exp \left(-x_{0}\right)$ is solved by $x_{0}=0.567$ and we thus have $\alpha R+\frac{1}{4} \alpha R A^{2}=x_{0}$, which is an implicit second degree equation for $\alpha$. The solutions are

$$
\alpha R=\frac{x_{0} \pm \sqrt{x_{0}^{2}-R^{2} / \xi^{2}}}{2} .
$$

The existance of a real solution is thus seen to require $R / \xi \leq x_{0}$. In addition, we see that $\alpha R=\frac{x_{0}}{2}$ for $R=x_{0} \xi$. However, we need to consider also the condition $\alpha \xi \ll$ $1\left(A^{2} \gg 1\right)$. which was a prerequisite for obtaining the eigenvalue equation in the form of Eq. (39). By expansion around $R / \xi \rightarrow 0$ of the roots in Eq. (40), one sees that the one with the minus sign goes to zero in this limit and must be discarded. The solution with the plus sign in Eq. (40) is shown in Fig. 2 of Ref. 32 as the solid (blue) curve. We have checked that for small $\alpha \xi$, a numerical solution of the integrals of Appendix $\mathrm{A}$ yields the same result.

A simple second order approximation around $R / \xi=0$, can be found from the acceptable solution with the plus sign in Eq. (40). Expansion yields

$$
\alpha R=x_{0}-\frac{1}{4 x_{0}}\left(\frac{R}{\xi}\right)^{2}+\mathrm{O}\left(R^{4} / \xi^{4}\right) .
$$


The second order result is shown as the dotted (black) line in Fig. 2 of Ref. 32 .

\section{Efimov Effect in the Born-Oppenheimer Limit}

For completeness of presentation and reference, in this appendix we discuss the Efimov effect in the heavy-heavylight system in the absence of a condensate background felt by the light particle. Following the Born-Oppenheimer description, this energy is now an effective potential for the two heavy particles as function of their distance $R$. So we have the heavy-heavy Schrödinger equation

$$
\left[-\frac{\hbar^{2}}{M} \nabla_{\boldsymbol{R}}^{2}+E_{R}(\boldsymbol{R})\right] \Phi(\boldsymbol{R})=E \Phi(\boldsymbol{R}),
$$

where the kinetic energy is missing a factor of 2 in the denominator since the reduced mass in the heavy-heavy system is $M / 2$. Here $E$ is the total energy of the system of light-heavy-heavy type. This equation can be massaged into a nicer form by defining $u(\boldsymbol{R})=R \Phi(\boldsymbol{R}), \kappa^{2}=$ $-M E / \hbar^{2}>0$ (we are looking for bound states with $E<$ $0)$, and $z=\kappa R$. This produces

$$
\frac{d^{2} u(z)}{d z^{2}}-u(z)+\frac{\beta(a, z)^{2}}{z^{2}} u(z)=0
$$

where

$$
\beta(a, z)^{2}=\frac{x_{0}^{2}}{2} \frac{M}{m}\left[1+\frac{1}{x_{0}^{2}\left(1+e^{x_{0}}\right)} \frac{R}{a}\right],
$$

is still a function of $z$. However, if we consider $z \ll \kappa a$ $(R \ll a)$ or $|a| \rightarrow \infty$, we can drop the second term and get a constant $\beta$. In this case, the solution to Eq. (43) which has the correct boundary condition at $z \rightarrow \infty$ (exponentially decreasing, $e^{-z}$ ) contains a modified Bessel of the second kind, $K_{n}(z)$. However, the order has to be imaginary, i.e. $n \rightarrow i\left(\beta^{2}-1 / 4\right)$, and we arrive at $u(z)=\sqrt{z} K_{i\left(\beta^{2}-1 / 4\right)}(z)$ (not normalized). In our case, $M \gg m$, so $\beta^{2} \gg 1$, i.e. it is always a positive imaginary order. The wave function $u(z)$ has the following behavior at small $z$

$$
u(z) \propto \sqrt{z} \cos \left(s_{0} \log (z)\right),
$$

where $s_{0}=\sqrt{\beta^{2}-1 / 4}$. This is the origin of the logperiodic behavior of the states found by Efimov when $|a| \rightarrow \infty$. In order to show this, we observe an interesting scaling invariance of Eq. (42); if we consider $\Phi(\lambda \boldsymbol{R})$, then it solves the Schrödinger equation with energy $\lambda^{2} E$ when $|a| \rightarrow \infty$ for $\lambda$ a real number. However, Eq. (45) further contrains the choice of $\lambda$. Using $z=\kappa R$, we have

$$
\Phi(\boldsymbol{R}) \propto \sqrt{\kappa R} \cos \left(s_{0} \log \left(\frac{R}{R_{0}}\right)\right),
$$

where $R_{0}$ is a necessary short-distance cut-off which comes from the repulsive cores of atoms at short distance. In order to preserve the $\lambda$ scaling, we need to require $s_{0} \log (\lambda)=$ $n \pi$, where $n$ is an integer. We immediately see that there is an infinitude of three-body bound states with energies

$$
E_{n}=E_{0} e^{-2 \pi n / s_{0}}
$$

which is the famous Efimov effect. The ground state is related to $R_{0}$ since $E_{0} \sim-\hbar^{2} / 2 M R_{0}^{2}$ (up to constants of order 1 that we have neglected for simplicity). For $M \gg m$, $s_{0}$ will be large and will therefore in turn lead to a small scale factor $e^{-2 \pi n / s_{0}}$, which means that the spectrum of three-body bound states is quite dense, there are many three-body bound states around! For fininte $a$, it is possible to show that the above considerations are good up to $R \sim|a|$, and that the number of bound states is approximately $N \sim s_{0} \log \left(|a| / R_{0}\right) / \pi$ and increases with $s_{0}$, i.e. with $\sqrt{M / m}$.

\section{References}

1. T. Kraemer et al., Nature 440, 315 (2006).

2. F. Ferlaino and R. Grimm, Physics 3, 9 (2010).

3. V. Efimov, Yad. Fiz 12, 1080 (1970); Sov. J. Nucl. Phys. 12, 589 (1970).

4. N. A. Baas et al., Physics of Atomic Nuclei 77, 336 (2014).

5. A. S. Jensen, K. Riisager, D. V. Fedorov, and E. Garrido, Rev. Mod. Phys. 76, 215 (2004).

6. E. Braaten and H.-W. Hammer, Phys. Rep. 428, 259 (2006).

7. Y. Nishida, Phys. Rev. A 79, 013629 (2009).

8. D. J. MacNeill and F. Zhou, Phys. Rev. Lett. 106, 145301 (2011).

9. N. G. Nygaard and N. T. Zinner, New J. Phys. 16, 023026 (2014).

10. J.-L. Song and F. Zhou, Phys. Rev. A 84, 013601 (2011).

11. P. Niemann and H.-W. Hammer, Phys. Rev. A 86, 013628 (2012).

12. S. Endo and M. Ueda, arXiv:1309.7797 (2013).

13. D. Borzov, M. S. Mashayekhi, S. Zhang, J.-L. Song, and F. Zhou, Phys. Rev. A 85, 023620 (2012).

14. F. Zhou and M. S. Mashayekhi, Ann. Phys., 328, 83 (2013).

15. M. S. Mashayekhi, J.-S. Bernier, D. Borzov, J.-L. Song, and F. Zhou, Phys. Rev. Lett. 110, 145301 (2013).

16. S.-J. Jiang, W.-M. Liu, G. W. Semenoff, and F. Zhou, arXiv:1307.4263 (2013).

17. D. Dasenbrook and A. Komnik, Phys. Rev. B 87, 094301 (2013).

18. S. P. Rath and R. Schmidt, Phys. Rev. A 88, 053632 (2013).

19. M. J. Bijlsma, B. A. Heringa, and H. T. C. Stoof, Phys. Rev. A 61, 053601 (2000).

20. L. Viverit, C. J. Pethick, and H. Smith, Phys. Rev. A 61 , 053605 (2000).

21. F. M. Cucchietti and E. Timmermans, Phys. Rev. Lett. 96, $210401(2006)$.

22. R. M. Kalas and D. Blume, Phys. Rev. A 73, 043608 (2006).

23. J. Tempere et al., Phys. Rev. B 80, 184504 (2009).

24. W. Casteels, J. Tempere, and J. T. Devreese, Phys. Rev. A 84, 063612 (2011). 
25. D. H. Santamore and E. Timmermans, New J. Phys. 13, 103029 (2011)

26. I. Affleck, A. W. W. Ludwig, and B. A. Jones, Phys. Rev. B 52, 9528 (1995).

27. J. L. Smith and Q. Si, Europhys. Lett. 45, 228 (1999).

28. A. M. Sengupta, Phys. Rev. B 61, 4041 (2000).

29. M. Vojta, C. Buragohain, and S. Sachdev, Phys. Rev. B 61, 15152 (2000).

30. G. Zaránd and E. Demler, Phys. Rev. B 66, 024427 (2002).

31. S. Florens, L. Fritz, and M. Vojta, Phys. Rev. Lett. 96 036601 (2006).

32. N. T. Zinner, EPL 101, 60009, (2013).

33. A. L. Fetter and J. D. Walecka: Quantum Theory of ManyParticle Systems (McGraw-Hill, San Francisco, 1971).

34. S. Tan, Ann. Phys. 323, 2952 (2008).

35. M. Valiente, Phys. Rev. A 85, 014701 (2012).

36. M. Berninger et al., Phys. Rev. Lett. 107, 120401 (2011).

37. P. Naidon, E. Hiyama, and M. Ueda, Phys. Rev. A 86, 012502 (2012).

38. C. Chin, arXiv:1111.1484 2 .

39. R. Schmidt, S. P. Rath, and W. Zwerger, Eur. Phys. J. B 85, 386 (2012).

40. J. Wang, J. P. D'Incao, B. D. Esry, and C. H. Greene, Phys. Rev. Lett. 108, 263001 (2012).

41. P. K. Sørensen, D. V. Fedorov, A. S. Jensen, and N. T. Zinner, Phys. Rev. A 86, 052516 (2012).

42. P. Naidon, S. Endo, and M. Ueda, Phys. Rev. Lett. 112, 105301 (2014).

43. Y. Wang, J. Wang, J. P. D'Incao, and C. H. Greene, Phys. Rev. Lett. 109, 243201 (2012).

44. P. K. Sørensen, D. V. Fedorov, A. S. Jensen, and N. T. Zinner, J. Phys. B: At. Mol. Opt. Phys. 46, 075301 (2013).

45. P. K. Sørensen, D. V. Fedorov, A. S. Jensen, and N. T. Zinner, Phys. Rev. A 88, 042518 (2013).

46. J. S. Borbely, R. van Rooij, S. Knoop, and W. Vassen, Phys. Rev. A 85, 022706 (2012)

47. S. Knoop, J. S. Borbely, W. Vassen, and S. J. J. M. F. Kokkelmans, Phys. Rev. A 86, 062705 (2012).

48. S. Knoop, private comminucation (2012).

49. M. Mudrich, S. Kraft, K. Singer, R. Grimm, A. Mosk, and M. Weidemüller, Phys. Rev. Lett. 88, 253001 (2002).

50. C. Morzok et al., Phys. Rev. A 79, 012717 (2009).

51. K. Enomoto, M. Kitagawa, K. Kasa, S. Tojo, and Y. Takahashi, Phys. Rev. Lett. 98, 203201 (2007).

52. M. Kitagawa et al., Phys. Rev. A 77, 012719 (2008).

53. C.-H. Wu, J. W. Park, P. Ahmadi, S. Will, and M. W. Zwierlein, Phys. Rev. Lett. 109, 085301 (2012).

54. T. Takekoshi et al., Phys. Rev. A 85, 032506 (2012).

55. C. Weber, S. John, N. Spethmann, D. Meschede, and A. Widera, Phys. Rev. A 82, 042722 (2010).

56. N. Spethmann, F. Kindermann, S. John, C. Weber, D. Meschede, and A. Widera, Appl. Phys. B 106, 13 (2012).

57. C. Chin, R. Grimm, P. S. Julienne, and E. Tiesinga, Rev. Mod. Phys. 82, 1225 (2010).

58. S. B. Papp et al., Phys. Rev. Lett. 101, 135301 (2008).

59. S. E. Pollack et al., Phys. Rev. Lett. 102, 090402 (2009).

60. N. Navon et al., Phys. Rev. Lett. 107, 135301 (2011).

61. R. J. Wild et al., Phys. Rev. Lett. 108, 145305 (2012).

62. P. Makotyn et al., Nature Phys. 10, 116 (2014).

63. L.-I. Ha et al., Phys. Rev. Lett. 110, 145302 (2013).

64. R. J. Fletcher et al., Phys. Rev. Lett. 111, 125303 (2013).

65. V. Makhalov, K. Martiyanov, and A. Turlapov, Phys. Rev. Lett. 112, 045301 (2014).
66. A. G. Sykes et al., Phys. Rev. A 89, 021601(R) (2014).

67. D. Hudson Smith, E. Braaten, D. Kang, and L. Platter, Phys. Rev. Lett. 112, 110402 (2014).

68. A. H. Castro Neto, F. Guinea, N. M. R. Peres, K. S. Novoselov, and A. K. Geim, Rev. Mod. Phys. 81, 109 (2009).

69. M. Z. Hasan and C. L. Kane, Rev. Mod. Phys. 82, 3045 (2010).

70. X.-L. Qi and S.-C. Zhang, Rev. Mod. Phys. 83, 1057 (2011).

71. A. V. Balatsky, I. Vekhter, and J.-X. Zhu, Rev. Mod. Phys. 78, 373 (2006).

72. H. Zhai, Int. J. Mod. Phys. 26, 1230001 (2012).

73. Z.-Y. Shi, X. Cui, and H. Zhai, Phys. Rev. Lett. 112, 013201 (2014).

74. Y. Nishida, S. Moroz, and D. T. Son, Phys. Rev. Lett. 110, 235301 (2013).

75. A. G. Volosniev, D. V. Fedorov, A. S. Jensen, and N. T. Zinner, arXiv:1312.6535 (2013). 